\title{
First Measurements with NeXtRAD, a Polarimetric X/L-band Radar Network
}

\author{
S. Alhuwaimel ${ }^{\dagger}$, S. Coetzee*, P. Cheng*, D. Du Plessis*, F. Fioranelli ${ }^{\ddagger}$, H. Griffiths ${ }^{\dagger}$, M.R. Inggs*, \\ D. Jordan*, W. Miceli ${ }^{\dagger}$, D. O’Hagan*, R. Palama ${ }^{\dagger}$ S. Paine*, M. Ritchie ${ }^{\dagger}$, J.S. Sandenbergh* A. Stevens* \\ *Department of Electrical Engineering \\ University of Cape Town, Rondebosch, South Africa \\ Email: Michael.Inggs@uct.ac.za \\ ${ }^{\dagger}$ Department of Electronic and Electrical Engineering, \\ University College London, London, United Kingdom \\ ${ }^{\ddagger}$ School of Engineering, University of Glasgow, Glasgow, United Kingdom
}

\begin{abstract}
NeXtRAD is a fully polarimetric, X/L-band sensor network. It is a development of the older NetRAD system and builds on the experience gained with extensive deployments of NetRAD for sea clutter and target measurements. In this paper we will report on the first measurements with NeXtRAD, looking primarily at sea clutter and some targets, as well as early attempts at calibration using corner reflectors, and an assessment of the polarimetric response of the system. We also highlight innovations allowing for efficient data manipulation after measurement campaigns, as well as the plans for the future with this system.
\end{abstract}

\section{INTRODUCTION}

Multistatic radar systems have been investigated in the radar community for a number of years. These systems consist of multiple transmitter and receiver nodes, measuring the diversity, in both target and clutter returns, obtained by varying the system geometry. This can offer potential advantages over conventional monostatic radar, such as enhanced target signatures and improved detection using multi-perspective views.Further, the receive-only passive nodes are less complex and more compact, hence suitable to be mounted on UAVs or other unmanned platforms for enhanced surveillance operations [1], [2].

The advantage of having multi-perspective views on the targets of interest and the possibility of modifying the deployment geometry if mobile nodes are available can be particularly beneficial for the identification, tracking, and classification of low RCS maritime targets such as small boats, inflatables, and periscopes. However, there are very limited available bistatic and multistatic sea clutter data, as the required systems and measurements to collect them are complex and often related to classified research. A significant challenge is posed by the need of synchronisng different nodes to the same, common phase reference to achieve coherency and good Doppler performance, even when the nodes are separated by baselines in the order of hundreds of metres or a few kilometres.

Coherent networks of sensors are becoming feasible, and the advantages of these sensors over conventional, monostatic radars needs to be fully understood. This requires a set of models for targets and clutter measured by these new sensors, so that performance of these systems can assessed during design.

One of the key objectives of this paper is to present the NeXtRAD as a tool for collaboration in the area of networks of fully polarimetric sensors to understand the underlying characteristics of clutter and targets when measured multistatically. The system is configured to be deployed in the open from small, self-contained, rugged cabinets.

In this paper we will present the latest measurements with the new the NeXtRAD system, based on field trials in Simon's Town (clutter and small targets) in December 2016. We also present the measurements made during laboratory integration of the system, as well as low power field measurements.

This paper starts with a review of the objectives of the system [2]. This is followed by a description of the system configuration (three nodes, one active, two passive) and the plans to calibrate it. We then present measurements already made during the development and preliminary testing phase, to be followed by the results from the December 2016 trials. The paper concludes with the proposed programme for the system over the five years after 2017.

\section{NeXtRAD OBJECTIVES}

A rationale for the NeXtRAD dual-band multistatic radar network, developed by University College London (UCL) and the University of Cape Town (UCT) was presented [2]. This radar is the successor to the NetRAD S-band system which was conceived as a flexible, low-cost testbed to make measurements of bistatic target and clutter signatures and to explore the issues of practical multistatic radar. In its initial form NetRAD consisted of a three-node network with a transmit power of $+23 \mathrm{dBm}$. The nodes were connected by cable for control and synchronisation, with a maximum cable length of $200 \mathrm{~m}$.

In $2010 / 11$ NetRAD was upgraded to provide $500 \mathrm{~W}$ transmit power and to remove the need for physical connection of the nodes, by using (Global Positioning System Disciplined Oscillators (GPSDOs) at each node and wireless links from the master node to each of the other nodes [3]. These modifications significantly increased the achievable range, and 
allowed much greater node separation. The key attribute of NetRAD is that it allows simultaneous measurement to be made of monostatic and bistatic signatures, and hence a direct comparison to be made. In this form NetRAD was used in trials in South Africa to make measurements of bistatic sea clutter and small maritime target signatures, and led to the important conclusion that bistatic sea clutter may be less "spiky" than the equivalent monostatic clutter [4]-[11].

NetRAD has also been used for measurements of the radar signatures of the microDoppler of human targets [12], wind turbines [13] and drones and birds [14], [15]. The experience gained with NetRAD has been incorporated in the design of the next-generation NeXtRAD radar, described in this paper. This is a three-node, dual-band (X-band and L-band) polarimetric multistatic radar. It has been designed to be more flexible, user-friendly and reliable.

\section{SyStem CONFIGURATION}

\section{A. High Level Description}

The system is comprised of a single active node and two passive nodes illustrated in Fig. 1. All three nodes are equipped with GPS disciplined oscillators [16] which supply a stable $100 \mathrm{MHz}$ reference clock to the digital and RF transceivers. Critical subsystems in each node can be monitored and controlled by a laptop computer. These three node computers are connected by $\mathrm{WiFi}$ links to a central command and control laptop $(\mathrm{C} \& \mathrm{C})$, which distributes an experiment header file to each node. Motorized pedestals equipped with GPS positioning software direct all antenna beams to a common target. Video recordings of the target scene are recorded by video cameras mounted on each receiver pedestal, providing a visual reference of the target scene during measurements.

\section{B. Digital Backend and REX}

Conversion between radio frequency and the $125 \mathrm{MHz}$ intermediate frequency is done by an RF transceiver system supplied by Reutech Radar, known as the Radar Exciter (REX). The Pentek Model 71621 software radio interface was procured for use as the digital transceiver in the active node of the radar. This module is equipped with three 16-bit ADC channels, and a 16-bit DAC output. Identical units are used in the passive nodes in receive-only mode.

\section{Database Management and Real Time Processing}

The raw radar data and corresponding ancillary data (e.g. video, audio, GPS, time, weather, sea state, log files) are stored in a Hierarchical Data File version 5 (HDF5) archive. They are stored in the untouched form so that they do not include any irreversible processing by-products. The keywords of the combined data are extracted and entered in to a relational database, in this case MySQL has been used. The database can then be used to search for specific characteristics or for common attributes.

A PHP web interface is planned for the future to make querying the database easier. A GPU pulse compressor has been developed to process the radar data further.
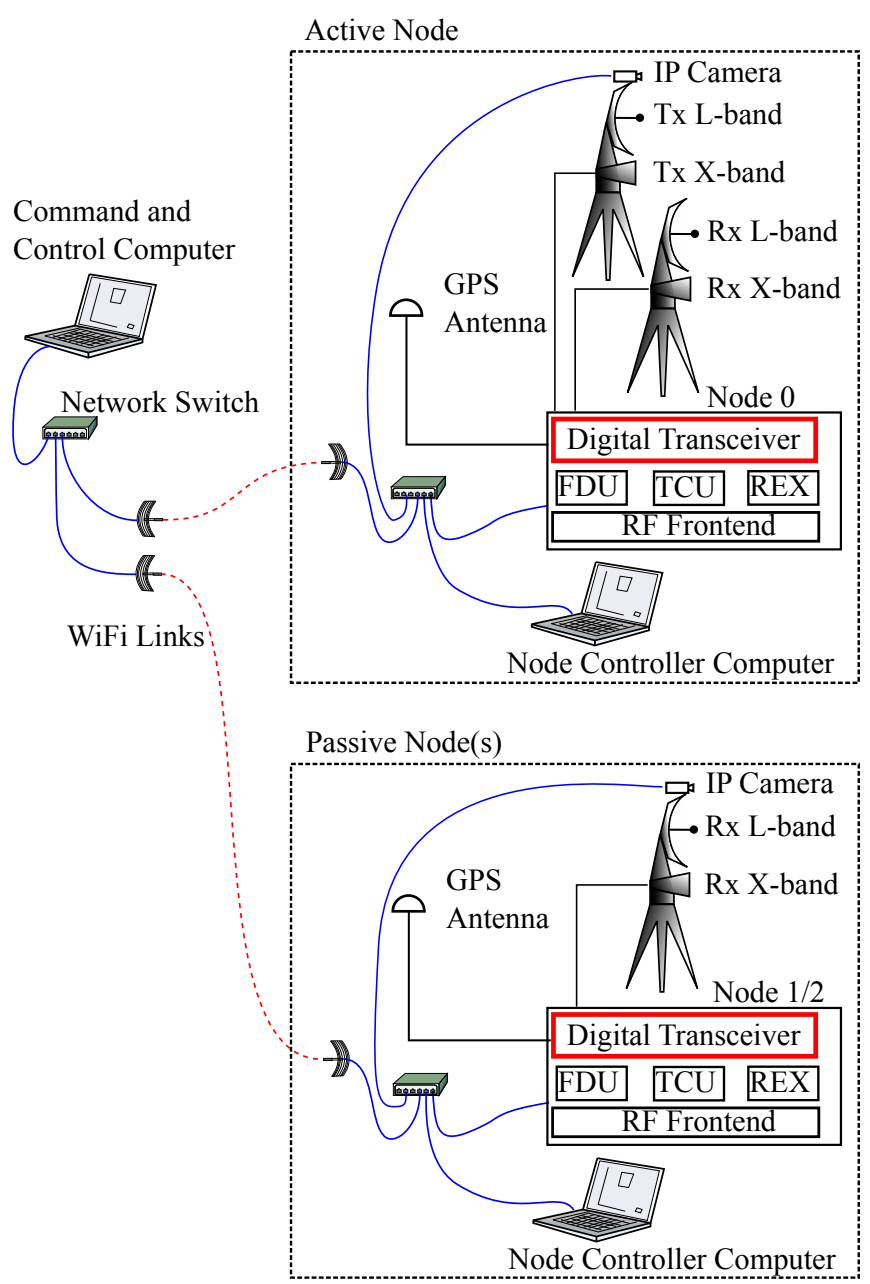

Fig. 1. The NeXtRAD system's configuration, showing an active/passive pair.

The recorded data are saved in binary format as sequential, 16-bit samples. Each range swath typically contained 2048 complex range samples, under a pulse repetition frequency of $1 \mathrm{kHz}$. Each range line would therefore need to be processed in under $1 \mathrm{~ms}$ if real-time plots are to be produced.

A quick-look processor was developed at UCT using popular freeware; FFTW and OpenCV. Using an off-the-shelf Intel i5-3750, real-time generation of range-time-intensity (RTI) and range-Doppler plots was achieved. A $60 \mathrm{~s}$ recording is processed in under $10 \mathrm{~s}$, significantly surpassing the real-time requirement, using the parameters outlined in Table I. The processor was tested using NetRAD data. The slow-quick time plot is shown in Fig. 2.

A multi-threaded version of the quick-look processor has been developed for NetRAD datasets and has been tested on a compute-oriented Amazon Web Services (AWS) instance. This provided a more than $10 \times$ acceleration over the singlethreaded implementation for large datasets, as shown in this paper. We intend to upgrade the NeXtRAD quick-look processor to a multi-threaded implementation and achieve a similar $10 \times$ speed-up. The convenience of having data and processing 


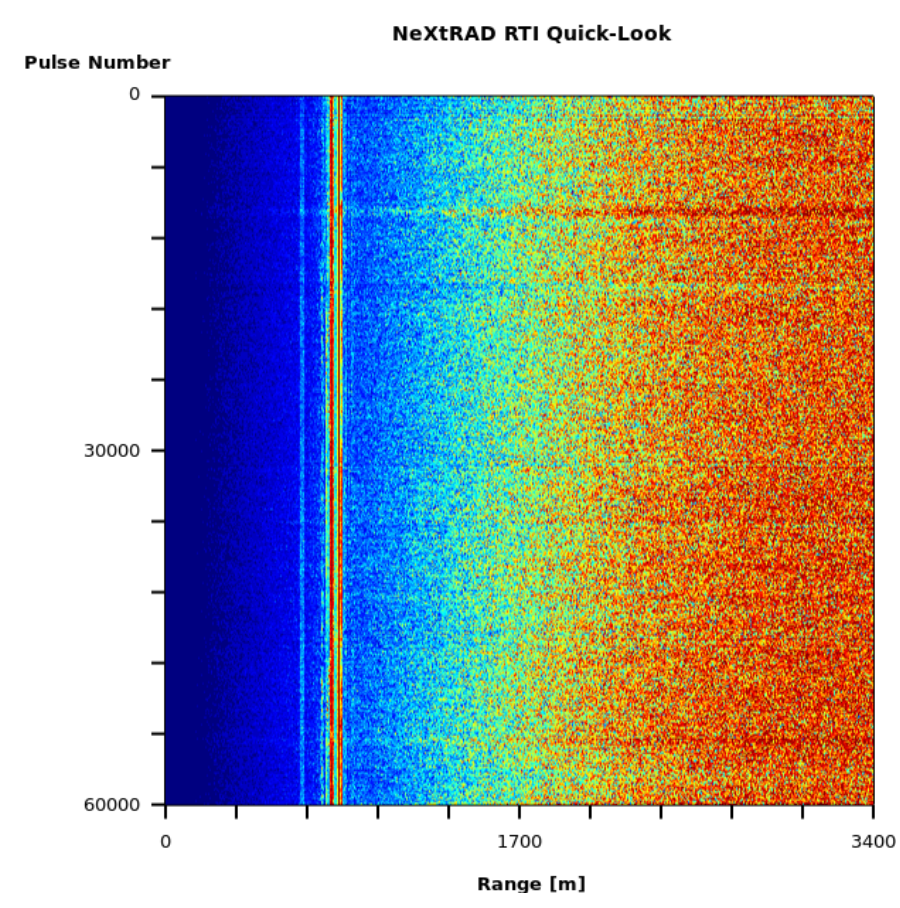

Fig. 2. Sample RTI Quick-Look using NetRAD data (S-band).

available publicly seems very attractive.

TABLE I

PRINCIPAL SET OF PROCESSING PARAMETERS.

\begin{tabular}{ll}
\hline Parameter & Value \\
\hline Range Lines in Data Set & 60000 \\
Complex Samples per Range Line & 2048 \\
Doppler FFT Size & 128 \\
Plot Updates during Processing & 100 \\
\hline
\end{tabular}

\section{Calibration Plans}

\section{A. $X$ - and L-Band Antennas}

The design and development of a dual polarised X-Band conical horn antenna (Fig. 3a) and an L-Band parabolic dish antenna (Fig. 3b) with centre frequencies of $8.5 \mathrm{GHz}$ and $1.3 \mathrm{GHz}$ respectively, with azimuth beamwidths of $10^{\circ}$ for both polarisations. The X-Band antenna is required to handle a peak power of $400 \mathrm{~W}$ while the L-Band antenna must be capable of $1.6 \mathrm{~kW}$, as well as low windage to allow mounting on a tripod.

An off-the-shelf antenna meeting the aforementioned specifications was either not available or unaffordable, and this has led the development of the antennas. The manufactured XBand antenna prototype was found to have an azimuth HPBW of $9.1^{\circ}$ and $10.4^{\circ}$ when horizontally and vertically polarised respectively. Similarly, the manufactured L-Band prototype antenna was found to have an azimuth HPBW $13.9^{\circ}$ and $12.4^{\circ}$ when horizontally and vertically polarised respectively. Detailed design procedure for X- and L-Band antennas are found in [17], [18] respectively.

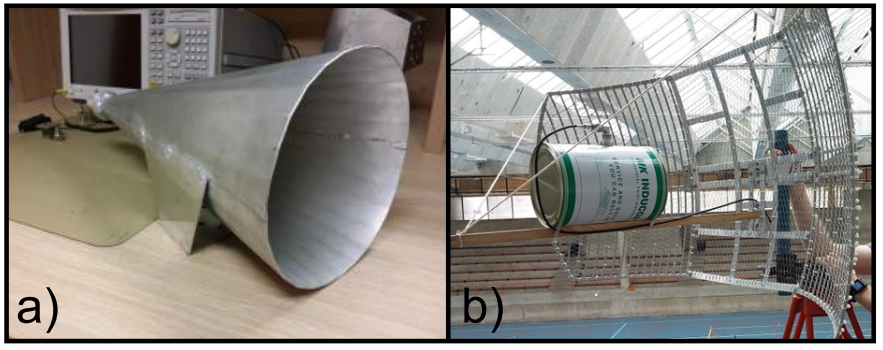

Fig. 3. Dual polarised antenna prototypes. a) X-Band conical horn antenna. b) L-Band parabolic dish antenna.

\section{B. Calibration Plans}

Imbalances and distortions exist in the radar system due to polarisation cross coupling, and power leakage between the orthogonal channels as well as between the nodes [19]. The distortions in the radar system can be found through the use of passive calibration targets. Calibration techniques are applied to find the distortion matrices, co- and cross-talk imbalances, isolation errors and phase errors. These distortions can be removed or minimized to correct the errors and non-idealities introduced by the antennas [20].

The triangular trihedral corner reflector is chosen for the initial monostatic measurements for NeXtRAD. The corner reflector provides large RCS but is very sensitive to radar alignment. The collected data can be analysed and calibration techniques can be performed to remove impurities in the data. Metallic spheres or cylindrical tubes are suitable for bistatic and multistatic measurements due to insensitivity to radar alignments but has low RCS compared to corner reflectors.

Alternatively, it is possible to build an active radar calibrator that provides the flexibility in configuring the bistatic angle and polarisation alignment. Active calibrators will not be implemented for the first NeXtRAD measurements.

\section{Measurements}

The NeXtRAD radar system has been preliminary tested in the laboratory at the University of Cape Town. Initially self-loop tests using a long section $(200 \mathrm{~m})$ of cable were performed to verify the effect of changing parameters of interest such as PRF and pulse length, as well as the phase stability in the Doppler domain through long recordings. Afterwards, additional tests were performed by locating the radar on the roof of the Department of Electrical Engineering at UCT and observing targets of interest such as cars and pedestrians in a nearby car park. X-band conical horn antennas described in Section IV-A were used to record one minute of data using a $50 \mathrm{MHz}$ bandwidth, $0.6 \mu$ s pulses at a $1 \mathrm{kHz}$ PRF on an $8.5 \mathrm{GHz}$ carrier. A pedestrian target and a slow moving car at approximately $75 \mathrm{~m}$ range were detected by microDoppler frequency shift, as seen in Fig. 4. These experiments have demonstrated the capability of the NeXtRAD radar to coherently capture range-time and Doppler signatures of these targets. 


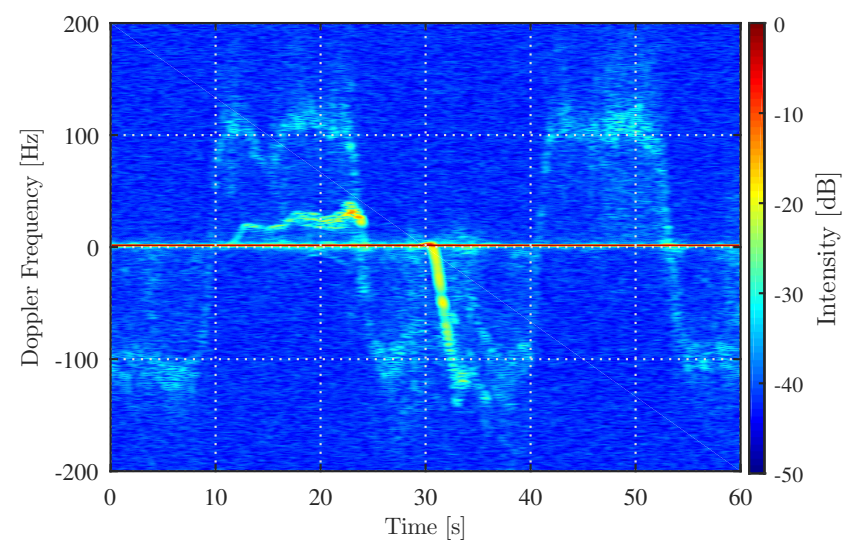

Fig. 4. A 60 second time time-Doppler plot of pedestrian target taken at a $1 \mathrm{kHz}$ PRF at X-band with the active node low-power prototype. Visible between 10 and 35 seconds in this plot is a strong return from a car moving out of a parking bay in the same area.

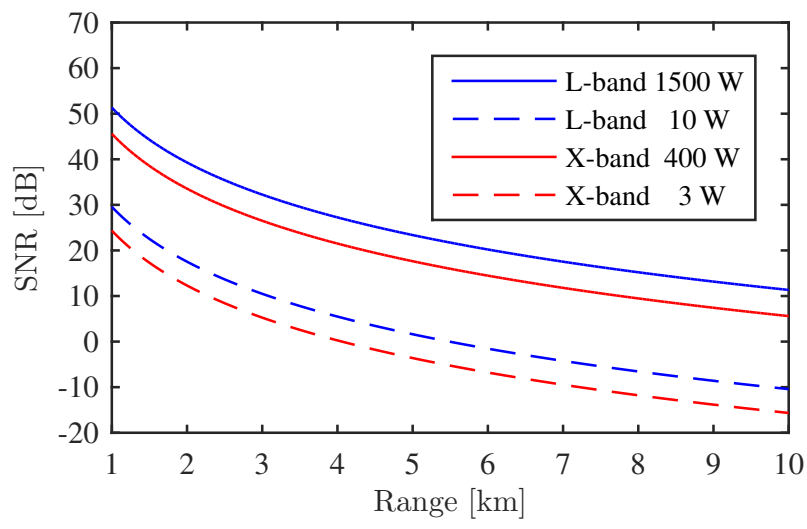

Fig. 5. Single pulse range vs SNR for a $1 \mathrm{~m} \times 1 \mathrm{~m}$ cylindrical target with standard system parameters using a $10 \mathrm{~W}$ and $1500 \mathrm{~W}$ L-band transmitter, and a $3 \mathrm{~W}$ and $400 \mathrm{~W} \mathrm{X}$-band transmitter.

Plots of the single-pulse range vs SNR using both of these transmitters is shown in Fig. 5. The modeled target is a $1 \mathrm{~m}$ radius, $1 \mathrm{~m}$ height metallic cylinder. Usable monostatic and bistatic calibration measurements were acquired with this modest transmitter power.

The key objectives for the NeXtRAD December 2016 measurement campaign were to gather multiband ( $\mathrm{L}$ and $\mathrm{X}$ ) dual polarized (at X-band) sea clutter data recorded over a series of azimuth angles and ranges, with respect to the wind and wave direction. The system was deployed on the roof of the South African Institute for Maritime Technology (IMT) building in Simon's Town. From this site, there was a clear $180^{\circ}$ of view over False Bay which enabled us to obtain data across a wide range of geometries. The scenario was a littoral environment and is not necessarily representative of an open sea, but this data will still be useful for the sea clutter research community.

The deployment location along with a series of example data capture geometries can be seen in Fig. 6. Meta data on the wind and wave conditions were recorded using local weather stations and buoys at sea. In particular, measurements were taken in monostatic geometry, by varying the azimuth pointing direction from $358^{\circ}$ then rotating clockwise to $58^{\circ}$, with a step of $10^{\circ}$. An interesting dataset was recorded on December 13, with high sea state conditions and a wind speed of about 20 knots. The transmitted signal was a LFM chirp with $50 \mathrm{MHz}$ bandwidth and $3 \mu$ s pulse length, modulated at three different carrier frequencies, $1.3 \mathrm{GHz}(\mathrm{L}), 8.5 \mathrm{GHz}(\mathrm{X} 1)$ and $9.2 \mathrm{GHz}$ (X2). A Pulse Repetition Frequency of $1 \mathrm{KHz}$ was used, and the total recording time was $120 \mathrm{~s}$, giving datasets of 120000 pulses.

Fig. 7 and 8 show the Range-Doppler maps of sea clutter data collected at an azimuth angle of $48^{\circ}$, horizontal polarization and a carrier frequency of $8.5 \mathrm{GHz}$ and $9.2 \mathrm{GHz}$. RangeDoppler maps were computed by using the average periodogram method, with windows of 256 pulses, normalizing the spectrum values on the maximum. Both figures show the zeroDoppler return from Roman Rock lighthouse located at about $1800 \mathrm{~m}$ from the radar. For the data collected at lower carrier frequency(Fig. 7), the clutter spectrum is included between $300 \mathrm{~m}$ and $800 \mathrm{~m}$ in range and between 0 and $-100 \mathrm{~Hz}$ in Doppler, with an average clutter-to-noise-ratio (CNR) of about $15 \mathrm{~dB}$, and a peak of about $20 \mathrm{~dB}$ at a range of $400 \mathrm{~m}$. Data collected at higher carrier frequency (Fig. 8) show a similar sea clutter spectrum, with a slightly smaller CNR, approximately $12 \mathrm{~dB}$. The presence of datasets collected at different carrier frequencies offer the opportunity to evaluate their impact on the sea clutter properties.

Measurements taken in this style include the CSIR 2007 $\mathrm{X}$-band database[21] that was generated from a position on Signal Hill, Cape Town. This dataset has proven to be a very useful asset for the research community and the goal of our measurement campaign is to expand on this using the additional degrees of freedom of frequency band and polarimetry. In order to allow for a close comparison of the $\mathrm{X}$ and L-band measured data the antennas will have the same beamwidth such that the illuminated patch is of comparable size in both cases. The main focus of the measurement campaign will be on sea clutter recordings, although additional target measurements may be taken at the time.

Within the configurable digital backend of the NeXtRAD radar node is a flexible arbitrary waveform generator (AWG) built into the Pentek ADC/DAC card. This will allow us to investigate the effects of waveform diversity on the sea clutter measurements that we take. Re-configuring both the waveform used and the central frequency of the system can be performed on a measurement-to-measurement basis. One of the interesting results that we would like to observe is the effect of range side-lobes from the match filtered pulse on the observed amplitude statistics of the sea clutter.

A further use of the AWG will be to transmit encoded data to produce a $50 \mathrm{MHz}$ noise like spectrum, and then test the reception and decoding of the data. This forms a symbiosis between the radar and a telecommunications system. 


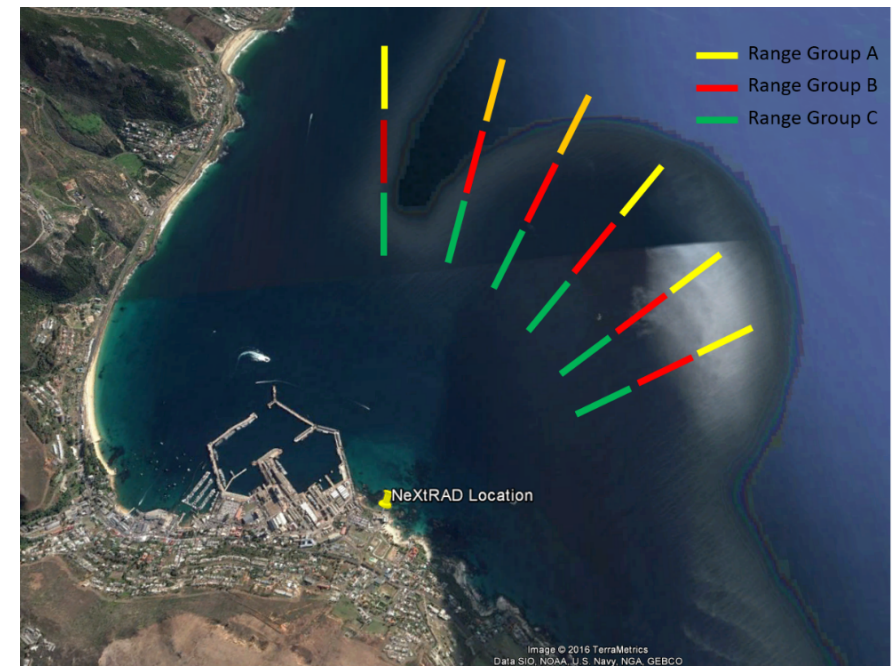

Fig. 6. NeXtRAD Deployment and measurement geometry.

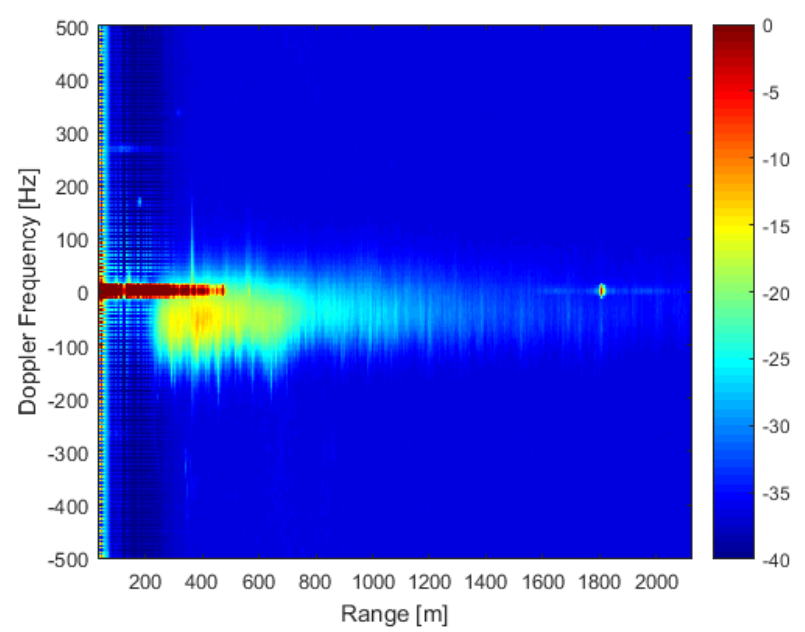

Fig. 7. Range-Doppler map of sea clutter data, collected at an azimuth angle of $48^{\circ}, 9.2 \mathrm{GHz}$ carrier frequency, horizontal polarization .

\section{PROgRAMme}

The system has been tested at low power (see Section V). The supporting subsystems incliding the data network between nodes, positioners, sound and video recording, L- and X-band polarimetric antennas, timing and frequency control within a node, and between nodes have all be tested and verified. Work now moves from the $3 \mathrm{~W}$ X-band and $20 \mathrm{~W}$ L-band power stages to higher power amplifiers to extend the detection range. The capability of following targets with low radial angular velocity, where the target is instrumented with GPS and a telemetry link has been successfully tested. The current antenna mounts are optical telescope mounts, not intended for high speed angular motion.

The system was deployed at the Institute for Maritime Studies in late November 2016, with a mostly monostatic set of sea clutter measurements being made at L- and Xband, including polarisation variations (Section $\mathrm{V}$ in the first

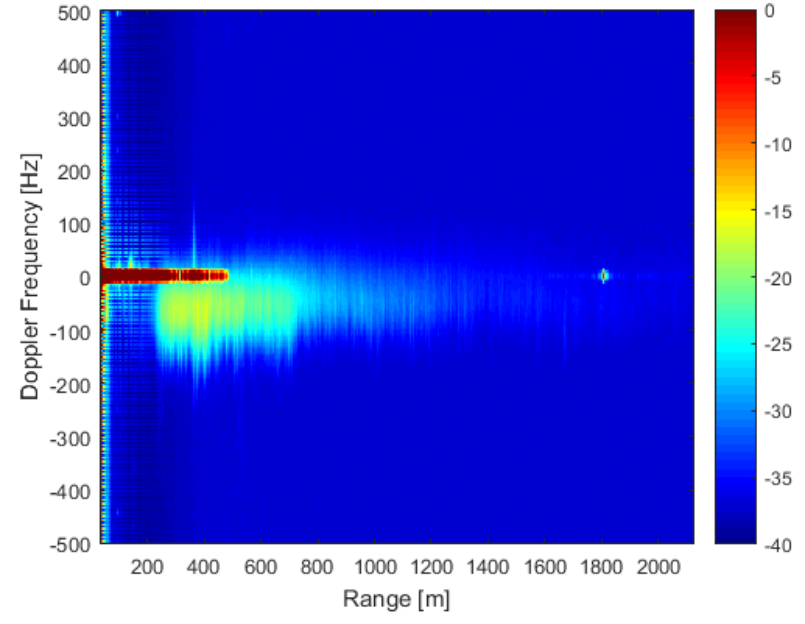

Fig. 8. Range-Doppler map of sea clutter data, collected at an azimuth angle of $48^{\circ}, 8.5 \mathrm{GHz}$ carrier frequency, horizontal polarization .

two weeks of December 2016. During these tests, we also evaluated the receive only nodes, albeit in a quasi monostatic configuration.

During the first quarter of 2017, the high power stages (1.5 kW at L-band and $400 \mathrm{~W}$ at X-band) will be integrated and some measurements repeated. The passive nodes will be integrated and first multistatic trials will be carried out. We will also address multistatic, polarimetric calibration, which is a difficult technical problem, and active calibrators seem to offer a flexible solution.

The system needs to be able to be deployed easily, and towards the end of 2017 the system will be decommissioned and rebuilt into quasi hermetic cabinets to allow for deployment close to the seaside for long periods while gathering data. Prototype enclosures with heat exchangers have been tested successfully.

We would like to encourage users world wide to use the system either in South Africa, or, transported to other sites. The complexity of the system will require at least one support person to be available, and operators will need to be trained. Hopefully this can be carried out collaboratively between the IMT and other potential users. Sharing of data and results should result, but can, of course, be protected by an appropriate joint agreement.

\section{CONCLUSIONS}

A successor to the S-band NetRAD has been described. We show that the low power loop back tests have been successful, achieving close to the $3 \mathrm{~m}$ pulse resolution expected of a $50 \mathrm{MHz}$ linear chirp. The waveform generators are completely programmable, so experimentation of pulse to pulse modulation changes can be accommodated. We then go on to describe the trials performed in December 2016 when medium power amplifiers were integrated ( $3 \mathrm{~W}$ at X-band and $10 \mathrm{~W}$ L-band). These tests were mostly monostatic, and conducted from the 
IMT building in Simon's Town, with Roman Rock lighthouse as a fiduciary target.

Plans for 2017 include mid year, full power tests in Simon's Bay, with the equipment housed in temporary shelters against weather. During 2018 the new, weatherproof, transportable cabinets will be introduced, to allow for flexible deployment, with a combination of generators and battery power supplies. We hope to see international users coming forward for collaborative trials.

\section{ACKNOWLEDGMENTS}

We gratefully acknowledge the SANDF for bursary funding over many years, and KACST for funding for S. Alhuwaimel. The ONR(G), FFI (Norway), IET AF Harvey Research Prize, NRF (South Africa) and EPSRC and the Royal Academy of Engineering have made substantial contributions to equipment and Postdoctoral funds. Staff at Armscor IMT (Simon's Town) have been very supportive.

\section{REFERENCES}

[1] N. J. Willis and H. D. Griffiths, Advances in bistatic radar. SciTech Publishing, 2007, vol. 2.

[2] M. Inggs, H. Griffiths, F. Fioranelli, M. Ritchie, and K. Woodbridge, "Multistatic radar: System requirements and experimental validation," in 2014 International Radar Conference, IEEE, 2014, pp. 1-6.

[3] J. S. Sandenbergh and M. R. Inggs, "A common view gpsdo to synchronize netted radar," in 2007 IET International Conference on Radar Systems, 2007.

[4] H. Griffiths, W. Al-Ashwal, K. Ward, R. Tough, C. Baker, and K. Woodbridge, "Measurement and modelling of bistatic radar sea clutter," IET radar, sonar \& navigation, vol. 4, no. 2, pp. 280-292, 2010.

[5] W. A. Al-Ashwal, A. Khenchaf, H. D. Griffiths, and K. Woodbridge, "Comparison between measurement and simulation of monostatic and bistatic sea clutter," in 2014 International Radar Conference, IEEE, 2014, pp. 1-6.

[6] M. Ritchie, A. Stove, K. Woodbridge, and H. Griffiths, "Netrad: Monostatic and bistatic sea clutter texture and doppler spectra characterization at s-band," IEEE Transactions on Geoscience and Remote Sensing, vol. 54, no. 9, pp. 5533-5543, 2016.

[7] F. Fioranelli, M. Ritchie, H. Griffiths, M. Inggs, and J. Sandenbergh, "Analysis of polarimetric bistatic sea clutter using the netrad radar system," IET Radar, Sonar and Navigation, 2016.

[8] W. A. Al-Ashwal, K. Woodbridge, and H. D. Griffiths, "Analysis of bistatic sea clutter - part ii: Amplitude statistics," IEEE Transactions on Aerospace and Electronic Systems, vol. 50, no. 2, pp. 1293-1303, Apr. 2014, ISSN: 0018-9251. DOI: 10 . 1109/TAES . 2014. 120434.
[9] — "Analysis of bistatic sea clutter - part i: Average reflectivity," IEEE Transactions on Aerospace and Electronic Systems, vol. 50, no. 2, pp. 1283-1292, Apr. 2014, ISSN: 0018-9251. DOI: 10 . 1109/TAES . 2014. 120426.

[10] M. Inggs, G. Inggs, J. Sandenbergh, W. Al-Ashwal, K. Woodbridge, and H. Griffiths, "Multistatic networked radar for sea clutter measurements," in 2011 IEEE International Geoscience and Remote Sensing Symposium, Jul. 2011, pp. 4449-4452. DOI: 10.1109/IGARSS.2011. 6050220 .

[11] W. A. Al-Ashwal, H. D. Griffiths, and K. Woodbridge, "An empirical model for bistatic sea clutter normalised radar cross section," in IET International Conference on Radar Systems (Radar 2012), Oct. 2012, pp. 1-5. DOI: 10.1049/cp.2012.1672.

[12] F. Fioranelli, M. Ritchie, and H. Griffiths, "Classification of unarmed/armed personnel using the netrad multistatic radar for micro-doppler and singular value decomposition features," IEEE Geoscience and Remote Sensing Letters, vol. 12, no. 9, pp. 1933-1937, 2015.

[13] M. Ritchie, F. Fioranelli, A. Balleri, and H. Griffiths, "Measurement and analysis of multiband bistatic and monostatic radar signatures of wind turbines," Electronics Letters, vol. 51, no. 14, pp. 1112-1113, 2015.

[14] F. Fioranelli, M. Ritchie, H. Griffiths, and H. Borrion, "Classification of loaded/unloaded micro-drones using multistatic radar," Electronics Letters, vol. 51, no. 22, pp. 1813-1815, 2015.

[15] M. Ritchie, F. Fioranelli, H. Griffiths, et al., "Monostatic and bistatic radar measurements of birds and microdrone," in 2016 IEEE Radar Conference (RadarConf), IEEE, 2016, pp. 1-5.

[16] J. S. Sandenbergh and M. R. Inggs, "A common view gpsdo to synchronize netted radar," in 2007 IET International Conference on Radar Systems, Oct. 2007, pp. 1-5. DOI: 10.1049/cp:20070499.

[17] P.-K. Cheng, "Nextrad antenna design: X-band dual polarised conical horn antenna,' Master's Dissertation, University of Cape Town, 2016.

[18] S. T. Paine, "Design and implementation of a dual polarised 1-band parabolic dish antenna for nextrad," Master's Dissertation, University of Cape Town, 2016.

[19] W. Wiesbeck and D. Kahny, "Single reference, three target calibration and error correction for monostatic, polarimetric free space measurements," Proceedings of the IEEE, vol. 79, no. 10, pp. 1551-1558, 1991.

[20] R. Palama', M. S. Greco, P. Stinco, and F. Gini, "Statistical analysis of bistatic and monostatic sea clutter," IEEE Transactions on Aerospace and Electronic Systems, vol. 51, no. 4, pp. 3036-3054, Oct. 2015, ISSN: 0018-9251. DOI: 10.1109/TAES.2015.140438.

[21] H. De Wind, J. Cilliers, and P. Herselman, "Dataware: Sea clutter and small boat radar reflectivity databases [best of the web]," IEEE Signal Processing Magazine, vol. 27, no. 2, pp. 145-148, 2010. 